\title{
„Ich halte es für unverzichtbar, offen über Fehler zu sprechen“
}

Auch ohne Präsenzveranstaltungen bleibt in Zeiten von COVID-19 die radiologische Fortbildung wichtig. Aus diesem Grund haben der Präsident der Rheinisch-Westfälischen Röntgengesellschaft Prof. Dr. Marco Das und Kongresspräsident Prof. Dr. Stefan Rohde die aktuellen Gegebenheiten zum Anlass genommen, den 13. RadiologieKongressRuhr (RKR) in den digitalen Raum zu überführen. Was Sie am 5. November 2020 in der virtuellen Spezialausgabe des RKR erwartet und was für eine zentrale Rolle dabei das Fehlermanagement spielt, berichten Prof. Das und Prof. Rohde im Interview.

Die COVID-19-Pandemie hat alle Bereiche des Lebens durcheinandergewirbelt auch den 13. RadiologieKongressRuhr. Eine komplette Absage wäre aus diesem Grund eine verständliche Lösung gewesen. Weshalb haben Sie sich für eine digitale Spezialausgabe des RKR entschieden?

Prof. Dr. Marco Das: Die Pandemie hat jeden Aspekt des gesellschaftlichen Lebens verändert. Ein persönlicher Austausch auf einem Kongress ist daher leider auf bisher unabsehbare Zeit nicht möglich. Dennoch ist das Thema Aus-, Fort- und Weiterbildung ein wichtiger Grundpfeiler in der Radiologie. Mit der digitalen Version des RKR wollen wir hierzu unseren Beitrag leisten. Wir haben versucht, einen abwechslungsreichen Mix zusammenzustellen, der für jeden etwas bietet: Assistentinnen und Assistenten, Fachärztinnen und -ärzte sowie sehr erfahrene Radiologinnen und Radiologen. Darüber hinaus wollen wir der Industrie eine Plattform bieten - denn
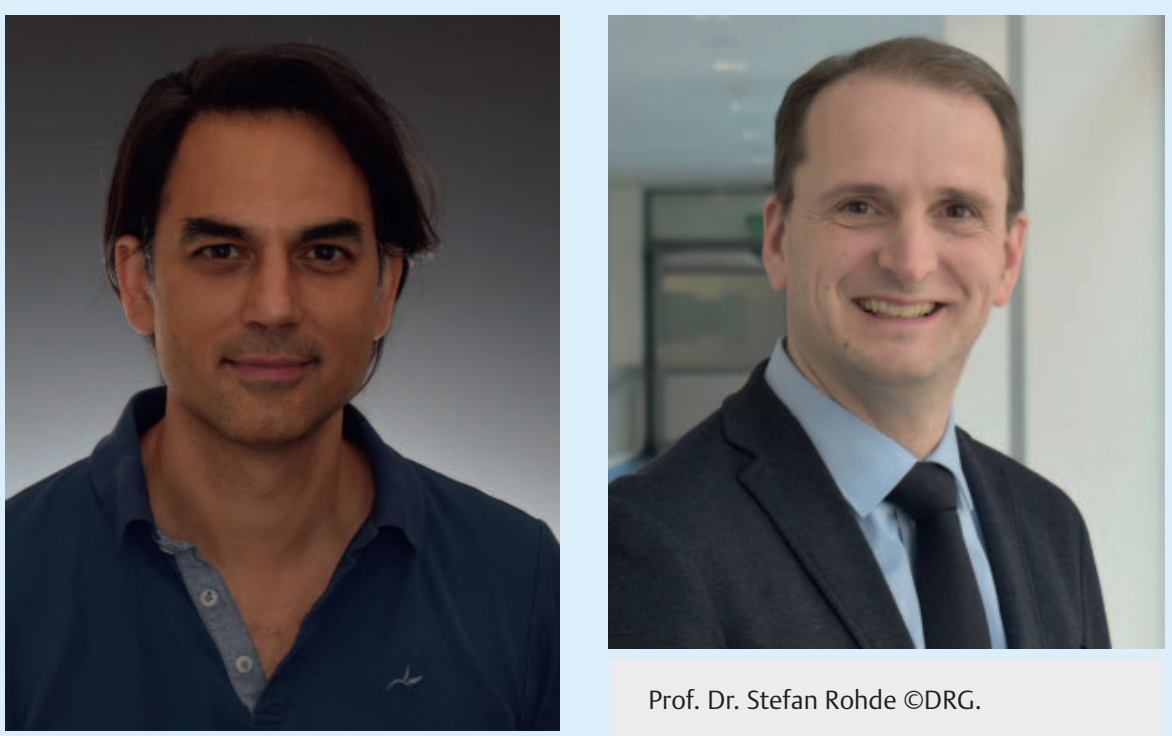

Prof. Dr. Stefan Rohde @DRG.

Prof. Dr. Marco Das @DRG.

auch diese Austauschmöglichkeit ist durch die Pandemie komplett weggefallen. Die Erfahrungen der letzten Monate mit einer Vielzahl von digitalen Konferenzen, Diskussionen und Fortbildungsprogrammen haben gezeigt, dass die digitalen Formate einen breiten Anklang finden, sodass ich mich sehr auf die digitale Version des RKR freue.

Prof. Dr. Stefan Rohde: Dem kann ich mich nur anschließen! Wir mussten in der aktuellen Situation den Kongress, so wie wir ihn ursprünglich geplant hatten, leider absagen. Das ist uns sehr schwergefallen. Durch das digitale Format haben wir nun die Möglichkeit, zumindest einen kleinen Ausschnitt des umfangreichen Fortbildungsprogramms des RKR darzustellen und einen Ausblick auf das nächste Jahr zu geben. Dann wird es hoffentlich möglich sein, den Kongress in regulärer Form zu organisieren.

Abdomenbildgebung, „Fit für den Facharzt", „Meine schönsten Fehler": Warum haben Sie gerade diese Themen und Formate für das virtuelle Weiterbildungsprogramm ausgewählt?

Das: Der RKR hat schon immer den Anspruch gehabt, ein breit aufgestellter Fortbildungskongress zu sein. Da wir nun eine verkürzte Form des Kongresses erstellt haben, mussten wir uns etwas wählerisch zeigen. Wir haben versucht, Themen herausder Radiologie ansprechen. Gleichzeitig sollen sich diese für verschiedenste Erfahrungslevel eignen. Insbesondere die „Fit für den Facharzt“-Kurse finden stets einen großen Anklang - sei es als Refresher oder als Vorbereitungskurs für den Facharzt. zugreifen, die eine breite Zuhörerschaft in 
Das Thema Fehlermanagement liegt mir sehr am Herzen. Warum? Ich halte es für unverzichtbar, offen über Fehler zu sprechen. Nicht um anzuklagen, sondern um zu lernen und zukünftige Fehler zu vermeiden. Wir müssen aufhören, mit dem Finger auf andere zu zeigen. Vielmehr müssen wir uns fragen: „Was haben wir aus dem Fehler gelernt und was können wir tun, damit wir den gleichen Fehler zukünftig nicht noch einmal machen?"

Rohde: Die Themenauswahl stellt eine „Miniaturausgabe“ des RKR dar: Zum einen wollten wir eine up to date Fortbildung für Fachärztinnen und Fachärzte sowie für nie- dergelassene Kolleginnen und Kollegen zu einem zentralen Thema der Radiologie anbieten - in diesem Fall die abdominelle Bildgebung. Zum anderen wollten wir auch wieder den Nachwuchs, insbesondere die Weiterbildungsassistentinnen und-assistenten kurz vor dem Facharzt, ansprechen - daher die FFF-Sitzung.

\section{Was ist Ihr persönliches Highlight im Rah- men der digitalen Spezialausgabe?}

Das: Es fällt mir schwer, etwas herauszugreifen, da alle Themen für sich genommen wichtig sind. Die Sitzung zum Fehlermanagement ist in gewisser Weise mein High- light, weil man eine offene Fehlerkultur nicht aus dem Buch lernen kann. Hilfreich ist es, aus den Fehlern anderer oder aus den eigenen Fehlern zu lernen. So können wir uns kontinuierlich verbessern.

Rohde: Die Auswahl stellt ja bereits eine Best-of-Ausgabe des RKR dar. Hiervon noch einen Favoriten zu benennen, ist praktisch unmöglich. Ich freue mich auf hervorragende Vorträge und spannende Diskussionen bei allen Sitzungen des digitalen RKR!

Danke Ihnen für das interessante Gespräch!

Sie sind neugierig geworden? Informationen zur Anmeldung und weitere Details erhalten Sie unter: www.radiologiekongressruhr.de

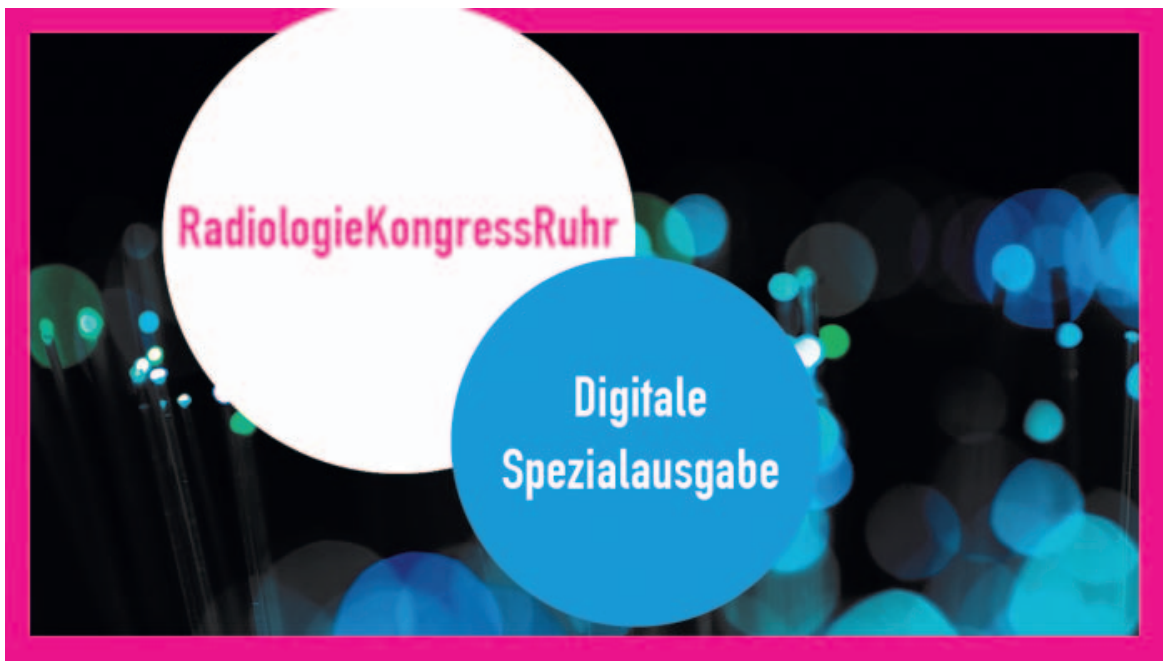

